\title{
Prolapse reduction deteriorates the urethral closure mechanism
}

\author{
Zi-Xi Loo ${ }^{1} \cdot$ Kun-Ling Lin $^{1,2} \cdot$ Cheng-Yu Long ${ }^{1,2,3}$ (B)
}

Received: 21 March 2019 / Accepted: 22 April 2019 /Published online: 14 May 2019

(C) The International Urogynecological Association 2019

\section{Dear Editor}

Yasmine Khayyami et al. have conducted great work in utilizing urethral pressure reflectometry (UPR), which is a novel instrument for detecting the urethral closure mechanism following pelvic organ prolapse (POP) [1]. Patients with POP may mask the existence of occult stress urinary incontinence (SUI) due to kinking of the urethra and obstruction from the advanced prolapse. Therefore, preoperative urodynamic assessment with prolapse reduction helps to detect postoperative SUI.

There is no general consensus on the standard method of prolapse reduction during urodynamic examination. A variety of methods have been used in prolapse reduction, such as large cotton swab, ring forceps, pessary, split speculum, and vaginal packing $[2,3]$. The instrument used for prolapse reduction in this study was a standard gynecological speculum. It was placed over the posterior vaginal wall with force exerted upon it during prolapse reduction, regardless of the prolapse compartment [1].

Anterior compartment POP has a greater impact on voiding function than apical or posterior compartment prolapse, which may be due to the kinking of the urethra and the pressure exerted by a cystocele on the urethra through a buttressing

Cheng-Yu Long

urolong@yahoo.com.tw

1 Department of Obstetrics and Gynecology, Kaohsiung Medical University Hospital, Kaohsiung Medical University, 100 Tzyou 1st Road, San-ming Dist, 807 Kaohsiung, Taiwan

2 Graduate Institute of Medicine, College of Medicine, Kaohsiung Medical University, Kaohsiung, Taiwan

3 Department of Obstetrics and Gynecology, Kaohsiung Municipal Hsiao-Kang Hospital, Kaohsiung Medical University, Kaohsiung, Taiwan effect. For those patients with anterior compartment prolapse, the use of this standard gynecological speculum may not truly mimic the postoperative prolapse reduction conditions as it was placed over the posterior vaginal wall. The final result of this study revealed that the number of positive stress tests increased from $4(18 \%)$ to $8(36 \%)$ in women with anterior compartment prolapse, whereas there was a greater increase in the positive stress tests from $1(6 \%)$ to $9(56 \%)$ in patients with posterior vaginal wall prolapse. The overcorrection of the posterior vaginal wall by placing a speculum for prolapse reduction in patients with solely anterior vaginal wall prolapse may further deteriorate urethral closure pressure in this study. We hope that this letter will deliver the message that there are various prolapse reduction techniques with proper selection so that more precise preoperative urodynamic assessment can be achieved.

\section{Compliance with ethical standards}

Conflicts of interest None.

\section{References}

1. Khayyami Y, Lose G, Klarskov N. Prolapse reduction deteriorates the urethral closure mechanism. Int Urogynecol J. 2019;30:489-93.

2. Visco AG, Brubaker L, Nygaard I, Richter HE, Cundiff G, Fine P, et al. The role of preoperative urodynamic testing in stress-continent women undergoing sacrocolpopexy: the Colpopexy and urinary reduction efforts (CARE) randomized surgical trial. Int Urogynecol J Pelvic Floor Dysfunct. 2008;19(5):607-14.

3. Ghoniem GM, Walters F, Lewis V. The value of the vaginal pack test in large cystoceles. J Urol. 1994;152:931-4.

Publisher's note Springer Nature remains neutral with regard to jurisdictional claims in published maps and institutional affiliations. 